\title{
LEIBNIZ COHOMOLOGY AND CONNECTIONS ON DIFFERENTIABLE MANIFOLDS
}

\author{
JERRY M. LODDER
}

\begin{abstract}
We show how an affine connection on a Riemannian manifold occurs naturally as a cochain in the complex for Leibniz cohomology of vector fields with coefficients in the adjoint representation. The Leibniz coboundary of the Levi-Civita connection can be expressed as a sum of two terms, one the Laplace-Beltrami operator and the other a Ricci curvature term. The vanishing of this coboundary has an interpretation in terms of eigenfunctions of the Laplacian. Additionally, we compute the Leibniz cohomology with adjoint coefficients for a certain family of vector fields on Euclidean $\mathbf{R}^{n}$ corresponding to the affine orthogonal Lie algebra, $n \geq 3$.
\end{abstract}

MSC Classification: 17A32, 53B05.

Key Words: Leibniz Cohomology, Levi-Civita Connection, Laplace Operator, Affine Orthogonal Lie Algebra, Eigenfunctions of the Laplacian.

\section{INTRODUCTION}

We study Leibniz cohomology, $H L^{*}$, of a Lie algebra $\mathfrak{g}$ with coefficients in its adjoint representation, written $H L^{*}(\mathfrak{g} ; \mathfrak{g})$, as a setting for capturing an affine connection [3, Chapter 2] as a cochain in the Leibniz complex $C L^{*}(\mathfrak{g} ; \mathfrak{g})=\operatorname{Hom}_{\mathbf{R}}\left(\mathfrak{g}^{\otimes *}, \mathfrak{g}\right)$. More specifically, let $M$ be a (real) differentiable manifold and $C^{\infty}(M)$ the ring of realvalued differentiable functions on $M$. Let $\chi(M)$ be the Lie algebra of differentiable vector fields on $M$. An affine or Koszul connection $\nabla$ on $M$ is an $\mathbf{R}$-linear map

$$
\nabla: \chi(M) \underset{\mathbf{R}}{\otimes} \chi(M) \rightarrow \chi(M)
$$

that is $C^{\infty}(M)$-linear in the first (left) tensor factor, but a derivation on $C^{\infty}(M)$ functions in the second (right) tensor factor. For $X, Y \in \chi(M)$, we write $\nabla(X \otimes Y)=$ $\nabla_{X}(Y)$. For $f \in C^{\infty}(M)$, we have

$$
\nabla_{X}(f Y)=X(f) Y+f \nabla_{X}(Y) .
$$

The failure of $\nabla$ to be $C^{\infty}(M)$ linear in its second $\chi(M)$ factor precludes $\nabla$ from being a two-tensor. In $\S 2$ we interpret $\delta \nabla$, the Leibniz coboundary of $\nabla$, in terms of the Laplace-Beltrami operator and the curvature operator $R: \chi(M)^{\otimes 3} \rightarrow \chi(M)$.

Mathematical Sciences, Deptartment 3MB; New Mexico State University; Las Cruces, NM, 88003; USA, jlodder@nmsu.edu. 
The vanishing of $\delta \nabla$ has an interpretation in terms of eigenfunctions of the Laplacian on manifolds of constant scalar curvature, Theorem (2.7).

Leibniz cohomology with coefficients in the adjoint representation for semi-simple Leibniz algebras has been studied in [6], while $H L^{*}(\mathfrak{L} ; \mathfrak{L})$ has been used to study deformation theory in the category of Leibniz algebras [4], 5]. Recall that a left Leibniz algebra $\mathfrak{L}$ over a commutative ring $k$ is a $k$-module equipped with a bilinear operation $\circ: \mathfrak{L} \times \mathfrak{L} \rightarrow \mathfrak{L}$ that is a derivation from the left:

$$
x \circ(y \circ z)=(x \circ y) \circ z+y \circ(x \circ z), \quad x, y, z \in \mathfrak{L} .
$$

There is a similar notion for a right Leibniz algebra [10]. A Lie algebra $\mathfrak{g}$ with its skew-symmetric bracket is both a left and a right Leibniz algebra, with the derivation property subsumed in the Jacobi identity. When working with Lie algebras in this paper, we will denote the Lie bracket as [, ] instead of o. Leibniz algebras were called $D$-algebras by Bloh [2] and later rediscovered and studied extensively by Loday [9, $\S 10.6 .1]$.

Since the bracket of a Lie algebra $\mathfrak{g}$ is skew-symmetric, there is more flexibility in stating the cochain complex yielding $H L^{*}(\mathfrak{g} ; \mathfrak{g})$ than for a (left or right) Leibniz algebra [6] [10]. Let

$$
C L^{n}(\mathfrak{g} ; \mathfrak{g})=\operatorname{Hom}_{k}\left(\mathfrak{g}^{\otimes n}, \mathfrak{g}\right), \quad n \geq 0,
$$

where $\mathfrak{g}$ is a Lie algebra over the commutative ring $k$. For calculations in this paper, $k=\mathbf{R}$. Let $\mathfrak{g}$ act on itself via the adjoint representation. For $g \in \mathfrak{g}$, the linear map $\operatorname{ad}_{g}: \mathfrak{g} \rightarrow \mathfrak{g}$ is given by

$$
g \cdot x:=\operatorname{ad}_{g}(x)=[g, x], \quad x \in \mathfrak{g} .
$$

Then $H L^{*}(\mathfrak{g} ; \mathfrak{g})$ is the cohomology of the cochain complex $C L^{*}(\mathfrak{g} ; \mathfrak{g})$ :

$$
\begin{aligned}
& \operatorname{Hom}_{k}(k, \mathfrak{g}) \stackrel{\delta}{\longrightarrow} \operatorname{Hom}_{k}(\mathfrak{g}, \mathfrak{g}) \stackrel{\delta}{\longrightarrow} \operatorname{Hom}_{k}\left(\mathfrak{g}^{\otimes 2}, \mathfrak{g}\right) \stackrel{\delta}{\longrightarrow} \operatorname{Hom}_{k}\left(\mathfrak{g}^{\otimes 3}, \mathfrak{g}\right) \stackrel{\delta}{\longrightarrow} \\
& \ldots \stackrel{\delta}{\longrightarrow} \operatorname{Hom}_{k}\left(\mathfrak{g}^{\otimes n}, \mathfrak{g}\right) \stackrel{\delta}{\longrightarrow} \operatorname{Hom}_{k}\left(\mathfrak{g}^{\otimes(n+1)}, \mathfrak{g}\right) \stackrel{\delta}{\longrightarrow} \ldots .
\end{aligned}
$$

For $f: \mathfrak{g}^{\otimes n} \rightarrow \mathfrak{g}$, the coboundary $\delta f: \mathfrak{g}^{\otimes(n+1)} \rightarrow \mathfrak{g}$ is given by

$$
\begin{aligned}
& (\delta f)\left(g_{1} \otimes g_{2} \otimes \ldots \otimes g_{n+1}\right)= \\
& \quad \sum_{i=1}^{n+1}(-1)^{i} g_{i} \cdot f\left(g_{1} \otimes g_{2} \otimes \ldots \widehat{g}_{i} \ldots \otimes g_{n+1}\right) \\
& \quad+\sum_{1 \leq i<j \leq n+1}(-1)^{j} f\left(g_{1} \otimes \ldots \otimes g_{i-1} \otimes\left[g_{i}, g_{j}\right] \otimes g_{i+1} \otimes \ldots \widehat{g}_{j} \ldots \otimes g_{n+1}\right) .
\end{aligned}
$$

In the next section we study $\delta \nabla$ for the Levi-Civita connection $\nabla$ on a differentiable manifold. In $\S 3$ we offer a calculation of $H L^{*}\left(\mathfrak{h}_{n} ; \mathfrak{h}_{n}\right)$, where $\mathfrak{h}_{n}$ is a certain family of vector fields on $\mathbf{R}^{n}$ isomorphic to the affine orthogonal Lie algebra, $n \geq 3$. This 
last section contains a subsection on the Pirashvili spectral sequence needed for the calculation of $H L^{*}$ as well as a subsection for the Lie-algebra cohomology of $\mathfrak{h}_{n}$ with adjoint coefficients.

\section{The Coboundary of the Levi-Civita Connection}

Let $M$ be an $n$-dimensional Riemannian manifold, $U \subseteq M$ an open subset of $M$, and $x: \mathbf{R}^{n} \stackrel{\cong}{\longrightarrow} U$ a chart in the $C^{\infty}$-structure on $M$. Then $\frac{\partial}{\partial x^{i}}, i=1,2, \ldots$, $n$ are local vector fields on $U$ with $\left[\frac{\partial}{\partial x^{i}}, \frac{\partial}{\partial x^{j}}\right]=0$. Let $\chi(M)$ be the Lie algebra of all (differentiable) vector fields on $M$. The Levi-Civita connection is the unique affine connection on $M$ that is symmetric and compatible with the Riemannian metric [3, Theorem 3.6]. For a symmetric connection $\nabla$ and $X, Y \in \chi(M)$, we have $\nabla_{X}(Y)-\nabla_{Y}(X)=[X, Y]$, and it follows that $\nabla_{\frac{\partial}{\partial x^{i}}}\left(\frac{\partial}{\partial x^{j}}\right)=\nabla_{\frac{\partial}{\partial x^{j}}}\left(\frac{\partial}{\partial x^{i}}\right)$. Let $\nabla(X \otimes Y)=\nabla_{X}(Y)$. In general, for any affine connection on $M$ and $X_{1}, X_{2}$, $X_{3} \in \chi(M)$, from Equation (1.3), we have

$$
\begin{aligned}
\delta \nabla\left(X_{1} \otimes X_{2} \otimes X_{3}\right)= & -\left[X_{1}, \nabla_{X_{2}}\left(X_{3}\right)\right]+\left[X_{2}, \nabla_{X_{1}}\left(X_{3}\right)\right]-\left[X_{3}, \nabla_{X_{1}}\left(X_{2}\right)\right] \\
& +\nabla_{\left[X_{1}, X_{2}\right]}\left(X_{3}\right)-\nabla_{\left[X_{1}, X_{3}\right]}\left(X_{2}\right)-\nabla_{X_{1}}\left(\left[X_{2}, X_{3}\right]\right) .
\end{aligned}
$$

Lemma 2.1. On Euclidean $\mathbf{R}^{1}$ let $\frac{d}{d x}$ be the canonical unit vector field for the identity chart. Let $X_{1}=f_{1} \frac{d}{d x}, X_{2}=f_{2} \frac{d}{d x}, X_{3}=f_{3} \frac{d}{d x}$ be vector fields, where $f_{1}, f_{2}, f_{3}: \mathbf{R} \rightarrow$ $\mathbf{R}$ are $C^{\infty}$ functions. Let $\nabla$ be the Levi-Civita connection. Then

$$
\delta \nabla\left(f_{1} \frac{d}{d x} \otimes f_{2} \frac{d}{d x} \otimes f_{3} \frac{d}{d x}\right)=\left(f_{1}^{\prime} f_{2} f_{3}^{\prime}-f_{1} f_{2}^{\prime} f_{3}^{\prime}-f_{1} f_{2} f_{3}^{\prime \prime}\right) \frac{d}{d x},
$$

where the latter can be interpreted as a certain alternating sum of terms in $\left(f_{1} f_{2} f_{3}^{\prime}\right)^{\prime}$.

Proof. In general $\left[f \frac{d}{d x}, g \frac{d}{d x}\right]=\left(f g^{\prime}-g f^{\prime}\right) \frac{d}{d x}$, and on Euclidean space

$$
\nabla_{f \frac{d}{d x}}\left(g \frac{d}{d x}\right)=f g^{\prime} \frac{d}{d x}
$$

From Equation (2.1), we have

$$
\begin{aligned}
& \delta \nabla\left(f_{1} \frac{d}{d x} \otimes f_{2} \frac{d}{d x} \otimes f_{3} \frac{d}{d x}\right)=-\left[f_{1} \frac{d}{d x}, f_{2} f_{3}^{\prime} \frac{d}{d x}\right]+\left[f_{2} \frac{d}{d x}, f_{1} f_{3}^{\prime} \frac{d}{d x}\right]-\left[f_{3} \frac{d}{d x}, f_{1} f_{2}^{\prime} \frac{d}{d x}\right] \\
& \quad+\nabla_{\left(f_{1} f_{2}^{\prime}-f_{2} f_{1}^{\prime}\right) \frac{d}{d x}}\left(f_{3} \frac{d}{d x}\right)-\nabla_{\left(f_{1} f_{3}^{\prime}-f_{3} f_{1}^{\prime}\right) \frac{d}{d x}}\left(f_{2} \frac{d}{d x}\right)-\nabla_{f_{1} \frac{d}{d x}}\left(f_{2} f_{3}^{\prime}-f_{3} f_{2}^{\prime}\right) \frac{d}{d x} .
\end{aligned}
$$

The result now follows by combining terms.

Thus, on $\mathbf{R}^{1}, \delta \nabla\left(\frac{d}{d x} \otimes \frac{d}{d x} \otimes f \frac{d}{d x}\right)=-f^{\prime \prime} \frac{d}{d x}$. Moving to $\mathbf{R}^{2}$ or to $\mathbf{R}^{n}$ in general, we recover the Laplacian of $f$ when used as a coefficient function on the third vector field. 
Lemma 2.2. On Euclidean $\mathbf{R}^{2}$, let $\frac{\partial}{\partial x}=\frac{\partial}{\partial x^{1}}$ and $\frac{\partial}{\partial y}=\frac{\partial}{\partial x^{2}}$ for the identity chart. Let $\nabla$ be the Levi-Civita connection and let $Z=c_{1} \frac{\partial}{\partial x}+c_{2} \frac{\partial}{\partial y}$, where $c_{1}, c_{2} \in \mathbf{R}$. Then

$$
\delta \nabla\left(\left(\frac{\partial}{\partial x} \otimes \frac{\partial}{\partial x}+\frac{\partial}{\partial y} \otimes \frac{\partial}{\partial y}\right) \otimes f Z\right)=-\left(f_{x x}+f_{y y}\right) Z .
$$

Proof. The proof follows from Lemma (2.1) or a direct calculation from the definition of $\delta$.

Corollary 2.3. For $Z=c_{1} \frac{\partial}{\partial x}+c_{2} \frac{\partial}{\partial y}$ a constant unit vector field on $\mathbf{R}^{2}$, i.e., $c_{1}^{2}+c_{2}^{2}=$ 1, we have

$$
\left\langle\delta \nabla\left(\left(\frac{\partial}{\partial x} \otimes \frac{\partial}{\partial x}+\frac{\partial}{\partial y} \otimes \frac{\partial}{\partial y}\right) \otimes f Z\right), Z\right\rangle=-\left(f_{x x}+f_{y y}\right)
$$

where $\langle$,$\rangle denotes the (Euclidean) metric on \mathbf{R}^{2}$.

The above lemma and corollary clearly generalize to $\mathbf{R}^{n}$.

For an arbitrary Riemannian manifold $M$, the $(1,3)$ curvature tensor of an affine connection $\nabla$ is given by $R: \chi(M)^{\otimes 3} \rightarrow \chi(M)$, where for $X, Y, Z \in \chi(M)$,

$$
R(X \otimes Y \otimes Z)=\nabla_{X} \nabla_{Y}(Z)-\nabla_{Y} \nabla_{X}(Z)-\nabla_{[X, Y]}(Z)
$$

using the sign convention from Sakai [13, p. 33]. We first state a general result that applies to any symmetric connection on a differentiable manifold.

Lemma 2.4. Let $M$ be a differentiable manifold with a symmetric connection $\nabla$. For $X, Z \in \chi(M)$, we have

$$
\delta \nabla(X \otimes X \otimes Z)=R(X \otimes Z \otimes X)-\nabla_{X} \nabla_{X}(Z)+\nabla_{\nabla_{X}(X)}(Z) .
$$

Proof.

$$
\begin{aligned}
\delta \nabla & (X \otimes X \otimes Z)=-\left[Z, \nabla_{X}(X)\right]-\nabla_{[X, Z]}(X)-\nabla_{X}([X, Z]) \\
& =-\nabla_{Z} \nabla_{X}(X)+\nabla_{\nabla_{X}(X)}(Z)-\nabla_{[X, Z]}(X)-\nabla_{X} \nabla_{X}(Z)+\nabla_{X} \nabla_{Z}(X) \\
& =\nabla_{X} \nabla_{Z}(X)-\nabla_{Z} \nabla_{X}(X)-\nabla_{[X, Z]}(X)-\nabla_{X} \nabla_{X}(Z)+\nabla_{\nabla_{X}(X)}(Z) \\
& =R(X \otimes Z \otimes X)-\nabla_{X} \nabla_{X}(Z)+\nabla_{\nabla_{X}(X)}(Z) .
\end{aligned}
$$

Since a connection is a local operator, we may express $\delta \nabla$ in terms of local coordinates, where an expression for the Laplacian may be more identifiable.

Corollary 2.5. Let $(x, U)$ be a coordinate chart for the $n$-dimensional Riemannian manifold $M$, let $Z \in \chi(M)$, and let $\nabla$ be the Levi-Civita connection on $M$. Then

$$
\begin{aligned}
& \delta \nabla\left(\sum_{i=1}^{n} \frac{\partial}{\partial x^{i}} \otimes \frac{\partial}{\partial x^{i}} \otimes Z\right)= \\
& \quad \sum_{i=1}^{n} R\left(\frac{\partial}{\partial x^{i}} \otimes Z \otimes \frac{\partial}{\partial x^{i}}\right)+\sum_{i=1}^{n}\left(-\nabla_{\frac{\partial}{\partial x^{i}}} \nabla_{\frac{\partial}{\partial x^{i}}}(Z)+\nabla_{\nabla_{\frac{\partial}{\partial x^{i}}}\left(\frac{\partial}{\partial x^{i}}\right)}(Z)\right) .
\end{aligned}
$$


Now, $\Delta(Z):=\sum_{i=1}^{n}\left(-\nabla_{\frac{\partial}{\partial x^{i}}} \nabla_{\frac{\partial}{\partial x^{i}}}(Z)+\nabla_{\nabla_{\frac{\partial}{\partial x^{i}}}\left(\frac{\partial}{\partial x^{i}}\right)}(Z)\right)$ is the Laplace-Beltrami operator applied to $Z$. We interpret $R$ in terms of Ricci curvature. Let \langle\rangle denote the metric on $M$, let $p \in M$, and let $z_{p}, y_{p} \in T_{p}(M)$ be tangent vectors to $M$ at $p$. For an orthonormal basis $\left\{e_{i}\right\}, i=1,2, \ldots, n$, of $T_{p}(M)$, the Ricci $(0,2)$ tensor is given by [13, p. 44]

$$
\operatorname{Ric}_{p}\left(z_{p}, y_{p}\right)=\sum_{i=1}^{n}\left\langle R\left(e_{i}, z_{p}, y_{p}\right), e_{i}\right\rangle=-\sum_{i=1}^{n}\left\langle R\left(e_{i}, z_{p}, e_{i}\right), y_{p}\right\rangle .
$$

Lemma 2.6. Let $(x, U)$ be a chart for the $n$-dimensional Riemannian manifold $(M,\langle\rangle)$ such that $e_{i}=\left.\frac{\partial}{\partial x^{i}}\right|_{p}, i=1,2, \ldots, n$, is an orthonormal basis for $T_{p}(M)$, $p \in U$. Let $Z \in \chi(M)$, and let $\nabla$ denote the Levi-Civita connection on $M$. If

$$
\delta \nabla\left(\sum_{i=1}^{n} \frac{\partial}{\partial x^{i}} \otimes \frac{\partial}{\partial x^{i}} \otimes Z\right)=0
$$

on $U$, then

for all $y \in T_{p}(M)$.

$$
\operatorname{Ric}_{p}(Z, y)=\langle\Delta(Z), y\rangle_{p}
$$

Proof. Since $\delta \nabla\left(\sum_{i=1}^{n} \frac{\partial}{\partial x^{i}} \otimes \frac{\partial}{\partial x^{i}} \otimes Z\right)=0$, we have $-\sum_{i=1}^{n} R\left(\frac{\partial}{\partial x^{i}} \otimes Z \otimes \frac{\partial}{\partial x^{i}}\right)=\Delta(Z)$. Thus, for any $y \in T_{p}(M)$, we have

$$
-\sum_{i=1}^{n}\left\langle R\left(\frac{\partial}{\partial x^{i}} \otimes Z \otimes \frac{\partial}{\partial x^{i}}\right), y\right\rangle_{p}=\langle\Delta(Z), y\rangle_{p}
$$

Since $\left\{\left.\frac{\partial}{\partial x^{i}}\right|_{p}\right\}$ is an orthonormal basis for $T_{p}(M)$, we have $\operatorname{Ric}_{p}(Z, y)=\langle\Delta(Z), y\rangle_{p}$ at the point $p$.

Theorem 2.7. With $(M,\langle\rangle)$ and $\nabla$ as in Lemma (2.6), suppose that $(x, U)$ is a geodesic normal coordinate chart around $p \in U$ with

$$
\delta \nabla\left(\sum_{i=1}^{n} \frac{\partial}{\partial x^{i}} \otimes \frac{\partial}{\partial x^{i}} \otimes f \frac{\partial}{\partial x^{j}}\right)=0
$$

on $U$ for $j=1,2, \ldots, n$, and a given $f \in C^{\infty}(M)$. Then $\Delta_{p} f=\left(\frac{s}{n}\right) f(p)$, where $s$ denotes the scalar curvature of $M$, and $\Delta$ is the Laplace-Beltrami operator (applied to $f)$.

Proof. In geodesic normal coordinates $\left\langle\frac{\partial}{\partial x^{i}}, \frac{\partial}{\partial x^{j}}\right\rangle_{p}=\delta_{i j}$, and $\nabla_{\left.\frac{\partial}{\partial x^{i}}\right|_{p}}\left(\frac{\partial}{\partial x^{j}}\right)=0$. Choose $y=\left.\frac{\partial}{\partial x^{j}}\right|_{p}$ in Lemma (2.6) . Then

$$
-\sum_{i=1}^{n}\left\langle R\left(\frac{\partial}{\partial x^{i}} \otimes f \frac{\partial}{\partial x^{j}} \otimes \frac{\partial}{\partial x^{i}}\right), \frac{\partial}{\partial x^{j}}\right\rangle_{p}=\left\langle\Delta\left(f \frac{\partial}{\partial x^{j}}\right), \frac{\partial}{\partial x^{j}}\right\rangle_{p} .
$$


In normal coordinates, $\Delta_{p}\left(f \frac{\partial}{\partial x^{j}}\right)=\sum_{i=1}^{n}\left(\frac{\partial^{2} f}{\partial x^{i} \partial x^{i}}\right)_{p} \frac{\partial}{\partial x^{j}}$. Thus, $\left\langle\Delta\left(f \frac{\partial}{\partial x^{j}}\right), \frac{\partial}{\partial x^{j}}\right\rangle_{p}=$ $\Delta_{p}(f)$. Since the curvature tensor $R$ is linear over $C^{\infty}(M)$, we have

$$
-f \sum_{i=1}^{n}\left\langle R\left(\frac{p}{\partial x^{i}} \otimes \frac{\partial}{\partial x^{j}} \otimes \frac{\partial}{\partial x^{i}}\right), \frac{\partial}{\partial x^{j}}\right\rangle_{p}=\Delta_{p}(f) .
$$

It follows that

$$
\begin{aligned}
& f(p) \cdot \operatorname{Ric}_{p}\left(\frac{\partial}{\partial x^{j}}, \frac{\partial}{\partial x^{j}}\right)=\Delta_{p}(f) \\
& f(p) \cdot s_{p}=f(p) \sum_{j=1}^{n} \operatorname{Ric}_{p}\left(\frac{\partial}{\partial x^{j}}, \frac{\partial}{\partial x^{j}}\right)=n \cdot \Delta_{p}(f) \\
& \Delta_{p}(f)=\left(\frac{s_{p}}{n}\right) f(p) .
\end{aligned}
$$

If the above holds for all $p \in M$, then globally $\Delta(f)=\left(\frac{s}{n}\right) f$. If furthermore $M$ is a manifold of constant scalar curvature $s_{p} \equiv c$, then $\Delta(f)=\left(\frac{c}{n}\right) f$, and $f$ is an eigenfunction of the Laplacian with eigenvalue $\frac{c}{n}$.

\section{Leibniz Cohomology of the Affine Orthogonal Lie Algebra}

We offer a calculation of $H L^{*}\left(\mathfrak{h}_{n} ; \mathfrak{h}_{n}\right)$, where $\mathfrak{h}_{n}$ is a certain family of vector fields on $\mathbf{R}^{n}$ isomorphic to the affine orthogonal Lie algebra, $n \geq 3$. Consider the standard coordinates on $\mathbf{R}^{n}$ given by $\left(x_{1}, x_{2}, \ldots, x_{n}\right)$ with unit vector fields $\frac{\partial}{\partial x^{i}}$ parallel to the $x_{i}$ axes. Let $\alpha_{i j}=x_{i} \frac{\partial}{\partial x^{j}}-x_{j} \frac{\partial}{\partial x^{i}}$. Then $\left\{\alpha_{i j}\right\}, 1 \leq i<j \leq n$, is a vector space basis for a Lie algebra isomorphic to $\mathfrak{s o}(n)$. Let $J_{n}$ be the $\mathbf{R}$ vector space spanned by $\left\{\frac{\partial}{\partial x^{i}}\right\}, i=1,2, \ldots, n$. Then $J_{n}$ is an Abelian Lie algebra. Let $\mathfrak{h}_{n}$ be the Lie algebra with basis given by the union of $\left\{\alpha_{i j}\right\}, 1 \leq i<j \leq n$, and $\left\{\frac{\partial}{\partial x^{i}}\right\}, i=1,2$, $\ldots, n$. Then $J_{n}$ is an ideal of $\mathfrak{h}_{n}$ and there is a short exact sequence of Lie algebras

$$
0 \longrightarrow J_{n} \longrightarrow \mathfrak{h}_{n} \longrightarrow \mathfrak{s o}(n) \longrightarrow 0
$$

with $\mathfrak{h}_{n} / J_{n} \simeq \mathfrak{s o}(n)$. We use results from invariant theory [1], where $H L^{*}\left(\mathfrak{h}_{n} ; \mathbf{R}\right)$ is computed, to help in the calculation of $H L^{*}\left(\mathfrak{h}_{n} ; \mathfrak{h}_{n}\right)$. In low dimensions $H L^{1}\left(\mathfrak{h}_{n} ; \mathfrak{h}_{n}\right)$ and $H L^{2}\left(\mathfrak{h}_{n} ; \mathfrak{h}_{n}\right)$ are generated by a "metric class" $I: \mathfrak{h}_{n} \rightarrow \mathfrak{h}_{n}$ and an "area class" $\rho: \mathfrak{h}_{n}^{\otimes 2} \rightarrow \mathfrak{h}_{n}$ respectively, where

$$
\begin{aligned}
& I\left(\frac{\partial}{\partial x^{i}}\right)=\frac{\partial}{\partial x^{i}}, \quad I\left(\alpha_{i j}\right)=0 \\
& \rho\left(\frac{\partial}{\partial x^{i}} \otimes \frac{\partial}{\partial x^{j}}\right)=\alpha_{i j}, \quad \rho\left(g_{1} \otimes g_{2}\right)=0 \text { if } g_{i} \in \mathfrak{s o}(n) .
\end{aligned}
$$

Higher dimensions of $H L^{*}\left(\mathfrak{h}_{n} ; \mathfrak{h}_{n}\right)$ contain echoes of these classes against a tensor algebra. Let $\alpha_{i j}^{*}$ be the dual of $\alpha_{i j}$ with respect to the basis $\left\{\alpha_{i j}\right\} \cup\left\{\frac{\partial}{\partial x^{i}}\right\}$ of $\mathfrak{h}_{n}$, and 
let $d x^{i}$ be the dual of $\frac{\partial}{\partial x^{i}}$. Let

$$
\gamma_{n}^{*}=\sum_{1 \leq i<j \leq n}(-1)^{i+j-1} \alpha_{i j}^{*} \otimes d x^{1} \wedge d x^{2} \wedge \ldots \widehat{d x^{i}} \ldots \widehat{d x^{j}} \ldots \wedge d x^{n}
$$

The element $\gamma_{n}^{*}: \mathfrak{h}_{n} \otimes J_{n}^{\wedge(n-2)} \rightarrow \mathbf{R}$ may be viewed as a factored volume form, which by skew-symmetry extends to $\mathfrak{h}_{n} \otimes \mathfrak{h}_{n}^{\otimes(n-2)} \rightarrow \mathbf{R}$. In subsection $\S 3.3$ we prove that

$$
H L^{*}\left(\mathfrak{h}_{n} ; \mathfrak{h}_{n}\right) \simeq\langle I, \rho\rangle \otimes T\left(\gamma_{n}^{*}\right)
$$

where $\langle I, \rho\rangle$ is the real vector space with basis $\{I, \rho\}$ and $T\left(\gamma_{n}^{*}\right):=\sum_{q \geq 0}\left\langle\gamma_{n}^{*}\right\rangle^{\otimes q}$ is the tensor algebra on the class of $\gamma_{n}^{*}$.

3.1. The Pirashvili Spectral Sequence. In this subsection we outline the cohomological version of the Pirashvili spectral sequence [12] for computing the Leibniz cohomology of a Lie algebra $\mathfrak{g}$ with coefficients in a $\mathfrak{g}$-module $V$, denoted $H L^{*}(\mathfrak{g} ; V)$. Suppose that both $\mathfrak{g}$ and $V$ are $k$-modules for a commutative ring $k$. We use the convention that $\mathfrak{g}$ acts on the left of $V$, meaning that for $g, h \in \mathfrak{g}$ and $x \in V$, we have $g(h x)-h(g x)=[g, h](x)$. Then $H L^{*}(\mathfrak{g} ; V)$ is the cohomology of the cochain complex $C L^{n}(\mathfrak{g} ; V)=\operatorname{Hom}_{k}\left(\mathfrak{g}^{\otimes n}, V\right), n=0,1,2,3, \ldots$ For $f \in C L^{n}(\mathfrak{g} ; V)$, $\delta f \in C L^{n+1}(\mathfrak{g} ; V)$ is given by Equation (1.3). If in Equation (1.3) the tensor product is replaced with the exterior product, then we have a cochain complex for $H_{\text {Lie }}^{*}(\mathfrak{g} ; V)$, the Lie-algebra cohomology of $\mathfrak{g}$ with coefficients in $V$. Using Pirashvili's grading, let $\pi_{\text {rel }}: \mathfrak{g}^{\otimes(n+2)} \rightarrow \mathfrak{g}^{\wedge(n+2)}, n \geq 0$, be the projection

$$
\pi_{\text {rel }}\left(g_{1} \otimes g_{2} \otimes \ldots \otimes g_{n+2}\right)=g_{1} \wedge g_{2} \wedge \ldots \wedge g_{n+2}
$$

There is an induced map on cohomology $\pi_{\text {rel }}^{*}: H_{\text {Lie }}^{*}(\mathfrak{g} ; V) \rightarrow H L^{*}(\mathfrak{g} ; V)$, and we study the relative groups. Let

$$
C_{\text {rel }}^{n}=\operatorname{Coker}\left[\operatorname{Hom}\left(\mathfrak{g}^{\wedge(n+2)}, V\right) \stackrel{\pi_{\text {rel }}}{\longrightarrow} \operatorname{Hom}\left(\mathfrak{g}^{\otimes(n+2)}, V\right)\right], \quad n=0,1,2, \ldots
$$

Then $C_{\text {rel }}^{*}$ is a cochain complex with cohomology denoted by $H_{\text {rel }}^{*}(\mathfrak{g} ; V)$. It follows quickly that $H_{\text {Lie }}^{0}(\mathfrak{g} ; V) \simeq H L^{0}(\mathfrak{g} ; V)$ and $H_{\text {Lie }}^{1}(\mathfrak{g} ; V) \simeq H L^{1}(\mathfrak{g} ; V)$. For higher dimensions there is a "Lie-to-Leibniz" long exact sequence:

$$
\begin{aligned}
0 \longrightarrow H_{\mathrm{Lie}}^{2}(\mathfrak{g} ; V) \stackrel{\pi_{\mathrm{rel}}^{*}}{\longrightarrow} H L^{2}(\mathfrak{g} ; V) \longrightarrow H_{\mathrm{rel}}^{0}(\mathfrak{g} ; V) \stackrel{c_{\mathrm{rel}}}{\longrightarrow} H_{\mathrm{Lie}}^{3}(\mathfrak{g} ; V) \longrightarrow \ldots \\
\longrightarrow H_{\mathrm{rel}}^{n}(\mathfrak{g} ; V) \stackrel{c_{\mathrm{rel}}}{\longrightarrow} H_{\mathrm{Lie}}^{n+3}(\mathfrak{g} ; V) \stackrel{\pi_{\mathrm{rel}}^{*}}{\longrightarrow} H L^{n+3}(\mathfrak{g} ; V) \longrightarrow H_{\mathrm{rel}}^{n+1}(\mathfrak{g} ; V) \longrightarrow \ldots
\end{aligned}
$$

Above, $c_{\text {rel }}$ is the connecting homomorphism. The Pirashvili spectral sequence arises from a filtration of $C_{\text {rel }}^{*}$ and converges to $H_{\text {rel }}^{*}(\mathfrak{g} ; V)$. 
First we describe $H_{\text {Lie }}^{*}\left(\mathfrak{g} ; \mathfrak{g}^{\prime}\right)$, the Lie-algebra cohomology of $\mathfrak{g}$ with coefficients in the coadjoint representation $\mathfrak{g}^{\prime}=\operatorname{Hom}_{k}(\mathfrak{g}, k)$. For $\varphi \in \mathfrak{g}^{\prime}$ and $g \in \mathfrak{g}$, the left action of $\mathfrak{g}$ on $\mathfrak{g}^{\prime}$ is given by

$$
(g \varphi)(x)=\varphi([x, g]), \quad x \in \mathfrak{g} .
$$

Then $H_{\text {Lie }}^{*}\left(\mathfrak{g} ; \mathfrak{g}^{\prime}\right)$ is the cohomology of the complex $\operatorname{Hom}_{k}\left(\mathfrak{g}^{\wedge *}, \mathfrak{g}^{\prime}\right)$. In general $H_{\text {Lie }}^{*}\left(\mathfrak{g} ; \mathfrak{g}^{\prime}\right)$ is not isomorphic to $H_{\text {Lie }}^{*}(\mathfrak{g} ; \mathfrak{g})$. However, $H_{\text {Lie }}^{*}\left(\mathfrak{g} ; \mathfrak{g}^{\prime}\right)$ can be computed from the isomorphic complex $\operatorname{Hom}_{k}\left(\mathfrak{g} \otimes \mathfrak{g}^{\wedge *}, k\right)$, which we now describe. Define $d: \mathfrak{g} \otimes \mathfrak{g}^{\wedge(n+1)} \rightarrow$ $\mathfrak{g} \otimes \mathfrak{g}^{\wedge n}, n=0,1,2, \ldots$, by

$$
\begin{aligned}
& d\left(x \otimes g_{1} \wedge g_{2} \wedge \ldots \wedge g_{n+1}\right)=\sum_{i=1}^{n+1}(-1)^{i+1}\left[x, g_{i}\right] \otimes g_{1} \wedge \ldots \widehat{g}_{i} \ldots \wedge g_{n+1} \\
& \quad+\sum_{1 \leq i<j \leq n+1}(-1)^{j+1} x \otimes g_{1} \wedge \ldots g_{i-1} \wedge\left[g_{i}, g_{j}\right] \wedge g_{i+1} \ldots \widehat{g}_{j} \ldots \wedge g_{n+1}
\end{aligned}
$$

The Lie-algebra homology groups $H_{*}^{\text {Lie }}(\mathfrak{g} ; \mathfrak{g})$ are computed from the complex $(\mathfrak{g} \otimes$ $\left.\mathfrak{g}^{\wedge *}, d\right)$. Let

$$
d^{*}: \operatorname{Hom}_{k}\left(\mathfrak{g} \otimes \mathfrak{g}^{\wedge n}, k\right) \rightarrow \operatorname{Hom}_{k}\left(\mathfrak{g} \otimes \mathfrak{g}^{\wedge(n+1)}, k\right)
$$

be the $\operatorname{Hom}_{k}$ dual of $d$, and let $\alpha \in \operatorname{Hom}_{k}\left(\mathfrak{g}^{\wedge n}, \mathfrak{g}^{\prime}\right)$. There is a cochain isomorphism

$$
\Phi: \operatorname{Hom}_{k}\left(\mathfrak{g}^{\wedge n}, \mathfrak{g}^{\prime}\right) \rightarrow \operatorname{Hom}_{k}\left(\mathfrak{g} \otimes \mathfrak{g}^{\wedge n}, k\right)
$$

given by

$$
\Phi(\alpha)\left(x \otimes g_{1} \wedge g_{2} \wedge \ldots \wedge g_{n}\right)=(-1)^{n} \alpha\left(g_{1} \wedge g_{2} \wedge \ldots \wedge g_{n}\right)(x)
$$

where $x, g_{1}, g_{2}, \ldots, g_{n} \in \mathfrak{g}$. The reader may verify that $\Phi(\delta \alpha)=d^{*}(\Phi \alpha)$.

Lemma 3.1. When $k$ is a field $\mathbf{F}$, we have

$$
H_{\text {Lie }}^{*}\left(\mathfrak{g} ; \mathfrak{g}^{\prime}\right) \simeq \operatorname{Hom}_{\mathbf{F}}\left(H_{*}^{\text {Lie }}(\mathfrak{g} ; \mathfrak{g}), \mathbf{F}\right)
$$

Proof. The proof follows from the existence of $\Phi$ and the universal coefficient theorem.

Consider now the projection

$$
\begin{aligned}
& \pi_{R}: \mathfrak{g} \otimes \mathfrak{g}^{\wedge(n+1)} \rightarrow \mathfrak{g}^{\wedge(n+2)}, \quad n \geq 0 \\
& \pi_{R}\left(g_{0} \otimes g_{1} \wedge g_{2} \wedge \ldots \wedge g_{n+1}\right)=g_{0} \wedge g_{1} \wedge g_{2} \wedge \ldots \wedge g_{n+1} .
\end{aligned}
$$

We work over a field $\mathbf{F}$, although many constructions are valid for an arbitrary commutative ring. Let

$$
C R^{n}(\mathfrak{g})=\operatorname{Coker}\left[\operatorname{Hom}_{\mathbf{F}}\left(\mathfrak{g}^{\wedge(n+2)}, \mathbf{F}\right) \stackrel{\pi_{R}^{*}}{\longrightarrow} \operatorname{Hom}_{\mathbf{F}}\left(\mathfrak{g} \otimes \mathfrak{g}^{\wedge(n+1)}, \mathbf{F}\right)\right]
$$


Then $C R^{*}(\mathfrak{g})$ is a cochain complex whose cohomology is denoted $H R^{*}(\mathfrak{g})$. We have $H_{\text {Lie }}^{1}(\mathfrak{g} ; \mathbf{F}) \simeq H_{\text {Lie }}^{0}\left(\mathfrak{g} ; \mathfrak{g}^{\prime}\right)$, and there is a "Lie-coadjoint" long exact sequence:

$$
\begin{aligned}
0 \longrightarrow H_{\text {Lie }}^{2}(\mathfrak{g} ; \mathbf{F}) \stackrel{\pi_{R}^{*}}{\longrightarrow} H_{\text {Lie }}^{1}\left(\mathfrak{g} ; \mathfrak{g}^{\prime}\right) \longrightarrow H R^{0}(\mathfrak{g}) \stackrel{c_{R}}{\longrightarrow} H_{\text {Lie }}^{3}(\mathfrak{g} ; \mathbf{F}) \longrightarrow \ldots \\
\longrightarrow H R^{m}(\mathfrak{g}) \stackrel{c_{R}}{\longrightarrow} H_{\text {Lie }}^{m+3}(\mathfrak{g} ; \mathbf{F}) \stackrel{\pi_{R}^{*}}{\longrightarrow} H_{\text {Lie }}^{m+2}\left(\mathfrak{g} ; \mathfrak{g}^{\prime}\right) \longrightarrow H R^{m+1}(\mathfrak{g}) \longrightarrow \ldots
\end{aligned}
$$

where $c_{R}$ is the connecting homomorphism. We are now ready to state the version of the Pirashvili spectral sequence used in this paper. Compare with [11] and [12].

Theorem 3.2. Let $\mathfrak{g}$ be a Lie algebra over a field $\mathbf{F}$ and let $V$ be a left $\mathfrak{g}$-module. Then there is a first-quadrant spectral sequence converging to $H_{\text {rel }}^{*}(\mathfrak{g} ; V)$ with

$$
E_{2}^{m, k} \simeq H R^{m}(\mathfrak{g}) \otimes H L^{k}(\mathfrak{g} ; V), \quad m \geq 0, \quad k \geq 0
$$

provided that $H R^{m}(\mathfrak{g})$ and $H L^{k}(\mathfrak{g} ; V)$ are finite dimensional vector spaces in each dimension. If this finiteness condition is not satisfied, then the completed tensor product $\widehat{\otimes}$ can be used.

Proof. We outline the key features of the construction and introduce notation that will be used in the sequel. Let $A^{m, k}$ denote those elements $f \in \operatorname{Hom}\left(\mathfrak{g}^{\otimes(k+m+2)}, V\right)$ that are skew-symmetric in the last $m+1$ tensor factors of $\mathfrak{g}^{\otimes(k+m+2)}$. Filter the complex $C_{\text {rel }}^{*}$ by

$$
F^{m, k}=A^{m, k} / \operatorname{Hom}\left(\Lambda^{k+m+2}(\mathfrak{g}), V\right) .
$$

Then $F^{m, *}$ is a subcomplex of $C_{\text {rel }}^{*}$ with $F^{0, *}=C_{\text {rel }}^{*}$ and $F^{m+1, *} \subseteq F^{m, *}$. To identify the $E_{0}^{*, *}$ term, use the isomorphism

$$
\operatorname{Hom}\left(\mathfrak{g}^{\otimes(k+m+2)}, V\right)=\operatorname{Hom}\left(\mathfrak{g}^{\otimes k} \otimes \mathfrak{g}^{\otimes(m+2)}, V\right) \simeq \operatorname{Hom}\left(\mathfrak{g}^{\otimes(m+2)}, \operatorname{Hom}\left(\mathfrak{g}^{\otimes k}, V\right)\right)
$$

Then

$$
\begin{aligned}
E_{0}^{m, k} & =F^{m, k} / F^{m+1, k-1} \\
& \simeq \operatorname{Hom}\left(\mathfrak{g} \otimes \mathfrak{g}^{\wedge(m+1)} / \mathfrak{g}^{\wedge(m+2)}, \operatorname{Hom}\left(\mathfrak{g}^{\otimes k}, V\right)\right),
\end{aligned}
$$

and $d_{m, k}^{0}: E_{0}^{m, k} \rightarrow E_{0}^{m, k+1}, m \geq 0, k \geq 0$. It follows that

$$
E_{1}^{m, k} \simeq \operatorname{Hom}\left(\mathfrak{g} \otimes \mathfrak{g}^{\wedge(m+1)} / \mathfrak{g}^{\wedge(m+2)}, H L^{k}(\mathfrak{g} ; V)\right) .
$$

Now, $d_{m, k}^{1}: E_{1}^{m, k} \rightarrow E^{m+1, k}$. Since the action of $\mathfrak{g}$ on $H L^{*}(\mathfrak{g} ; V)$ is trival, we have $E_{2}^{m, k} \simeq H R^{m}(\mathfrak{g}) \widehat{\otimes} H L^{k}(\mathfrak{g} ; V)$. Using the isomorphism (3.3), we consider an element of $E_{2}^{m, k}$ operationally in the form $H L^{k}(\mathfrak{g} ; V) \widehat{\otimes} H R^{m}(\mathfrak{g})$. 
3.2. Lie-Algebra Cohomology. For a Lie algebra $\mathfrak{g}$ and a left $\mathfrak{g}$-module $V$ over a field, let

$$
V^{\mathfrak{g}}=\{v \in V \mid g v=0, \forall g \in \mathfrak{g}\}
$$

be the subspace of invariants. Let $V_{\mathfrak{g}}=V /[\mathfrak{g}, V]$ be the quotient space of coinvariants, where $[\mathfrak{g}, V]$ is the span of all elements of the form $\{g v \mid g \in \mathfrak{g}, v \in V\}$. For $f \in \operatorname{Hom}\left(\mathfrak{g}^{\otimes n}, V\right)$ and $g \in \mathfrak{g}$, define the action of $\mathfrak{g}$ on $\operatorname{Hom}\left(\mathfrak{g}^{\otimes n}, V\right)$ by

$$
\begin{aligned}
& (g f)\left(x_{1} \otimes x_{2} \otimes \ldots \otimes x_{n}\right)=g \cdot f\left(x_{1} \otimes x_{2} \otimes \ldots \otimes x_{n}\right) \\
& \quad+\sum_{i=1}^{n} f\left(x_{1} \otimes \ldots \otimes x_{i-1} \otimes\left[x_{i}, g\right] \otimes x_{i+1} \otimes \ldots \otimes x_{n}\right), \quad x_{i} \in \mathfrak{g} .
\end{aligned}
$$

The action of $\mathfrak{g}$ on $\mathbf{R}$ is always considered trivial, meaning $g \cdot c=0, \forall g \in \mathfrak{g}$, $\forall c \in \mathbf{R}$, which determines the action of $\mathfrak{g}$ on $\operatorname{Hom}\left(\mathfrak{g}^{\otimes n}, \mathbf{R}\right)$. The above action on $\operatorname{Hom}\left(\mathfrak{g}^{\otimes n}, V\right)$ clearly induces an action on skew-symmetric elements of $\operatorname{Hom}\left(\mathfrak{g}^{\otimes n}, V\right)$. The resulting action of $\mathfrak{g}$ on $\operatorname{Hom}\left(\mathfrak{g}^{\wedge *}, V\right)$ commutes with the (Chevalley-Eilenberg) coboundary map $\delta$, and induces an action on $H_{\text {Lie }}^{*}(\mathfrak{g} ; V)$ [7]. Recall the definition of $\mathfrak{h}_{n}$ and $J_{n}$ given at the beginning of $\S 3$. In this subsection we compute $H_{\text {Lie }}^{*}\left(\mathfrak{h}_{n} ; \mathbf{R}\right)$, $H_{\text {Lie }}^{*}\left(\mathfrak{h}_{n} ; \mathfrak{h}_{n}\right)$, and $H_{\text {Lie }}^{*}\left(\mathfrak{h}_{n} ; \mathfrak{h}_{n}^{\prime}\right)$ by applying the Hochschild-Serre spectral sequence [7] and identifying certain $\mathfrak{s o}(n)$-invariant cochains. The calculations for $H R^{*}\left(\mathfrak{h}_{n}\right)$ follow from exact sequence (3.2).

Lemma 3.3. For $n \geq 3$, there are Hochschild-Serre isomorphisms in Lie-algebra cohomology

$$
\begin{aligned}
& H_{\mathrm{Lie}}^{*}\left(\mathfrak{h}_{n} ; \mathbf{R}\right) \simeq H_{\mathrm{Lie}}^{*}(\mathfrak{s o}(n) ; \mathbf{R}) \otimes\left[H_{\mathrm{Lie}}^{*}\left(J_{n} ; \mathbf{R}\right)\right]^{\mathfrak{s o}(n)} \\
& H_{\mathrm{Lie}}^{*}\left(\mathfrak{h}_{n} ; \mathfrak{h}_{n}\right) \simeq H_{\mathrm{Lie}}^{*}(\mathfrak{s o}(n) ; \mathbf{R}) \otimes\left[H_{\mathrm{Lie}}^{*}\left(J_{n} ; \mathfrak{h}_{n}\right)\right]^{\mathfrak{s o}(n)} \\
& H_{\mathrm{Lie}}^{*}\left(\mathfrak{h}_{n} ; \mathfrak{h}_{n}^{\prime}\right) \simeq H_{\mathrm{Lie}}^{*}(\mathfrak{s o}(n) ; \mathbf{R}) \otimes\left[H_{\mathrm{Lie}}^{*}\left(J_{n} ; \mathfrak{h}_{n}^{\prime}\right)\right]^{\mathfrak{s o}(n)}
\end{aligned}
$$

Proof. The proof follows by applying the Hochschild-Serre spectral sequence [7] to the ideal $J_{n}$ of $\mathfrak{h}_{n}$ and using the isomorphism of Lie algebras $\mathfrak{h}_{n} / J_{n} \simeq \mathfrak{s o}(n)$.

Now $\left[H_{\text {Lie }}^{*}\left(J_{n} ; \mathbf{R}\right)\right]^{\mathfrak{s o}(n)}$ is the cohomology of the cochain complex

$$
\left[\operatorname{Hom}\left(J_{n}^{\wedge *}, \mathbf{R}\right)\right]^{\mathfrak{s o}(n)} \simeq \operatorname{Hom}\left(\left(J_{n}^{\wedge *}\right)_{\mathfrak{s o}(n)}, \mathbf{R}\right) \simeq \operatorname{Hom}\left(\left(J_{n}^{\wedge *}\right)^{\mathfrak{s o}(n)}, \mathbf{R}\right) .
$$

From [1] or by a direct calculation

$$
\left(J_{n}^{\wedge k}\right)^{\mathfrak{s o}(n)}= \begin{cases}\mathbf{R} & k=0 \\ \left\langle\frac{\partial}{\partial x^{1}} \wedge \frac{\partial}{\partial x^{2}} \wedge \ldots \wedge \frac{\partial}{\partial x^{n}}\right\rangle & k=n \\ 0 & \text { otherwise. }\end{cases}
$$

Let $d x^{i}$ be the element of $\operatorname{Hom}\left(\mathfrak{h}_{n}, \mathbf{R}\right)$ dual to $\frac{\partial}{\partial x^{i}}$ with respect to the basis $\left\{\alpha_{i j}\right\} \cup$ $\left\{\frac{\partial}{\partial x^{i}}\right\}$ of $\mathfrak{h}_{n}$. 
Lemma 3.4. For $n \geq 3$,

$$
H_{\mathrm{Lie}}^{*}\left(\mathfrak{h}_{n} ; \mathbf{R}\right) \simeq H_{\mathrm{Lie}}^{*}(\mathfrak{s o}(n) ; \mathbf{R}) \oplus\left(H_{\mathrm{Lie}}^{*}(\mathfrak{s o}(n) ; \mathbf{R}) \otimes\left\langle v_{n}^{*}\right\rangle\right),
$$

where $v_{n}^{*}=d x^{1} \wedge d x^{2} \wedge \ldots \wedge d x^{n}$.

Likewise, $\left[H_{\text {Lie }}^{*}\left(J_{n} ; \mathfrak{h}_{n}^{\prime}\right)\right]^{\mathfrak{s o}(n)}$ is the cohomology of the cochain complex

$$
\left[\operatorname{Hom}\left(\mathfrak{h}_{n} \otimes J_{n}^{\wedge *}, \mathbf{R}\right)\right]^{\mathfrak{s o}(n)} \simeq \operatorname{Hom}\left(\left(\mathfrak{h}_{n} \otimes J_{n}^{\wedge *}\right)_{\mathfrak{s o}(n)}, \mathbf{R}\right) \simeq \operatorname{Hom}\left(\left(\mathfrak{h}_{n} \otimes J_{n}^{\wedge *}\right)^{\mathfrak{s o}(n)}, \mathbf{R}\right) .
$$

Now, $\left(\mathfrak{h}_{n} \otimes J_{n}^{\wedge *}\right)^{\mathfrak{s o}(n)} \simeq\left(J_{n} \otimes J_{n}^{\wedge *}\right)^{\mathfrak{s o}(n)} \oplus\left(\mathfrak{s o}(n) \otimes J_{n}^{\wedge *}\right)^{\mathfrak{s o}(n)}$. From [1], we have

$$
\left(J_{n} \otimes J_{n}^{\wedge k}\right)^{\mathfrak{s o}(n)} \simeq \begin{cases}\left\langle g_{n}\right\rangle & k=1 \\ \left\langle w_{n}\right\rangle & k=n-1 \\ 0 & \text { otherwise }\end{cases}
$$

where $g_{n}=\sum_{i=1}^{n} \frac{\partial}{\partial x^{i}} \otimes \frac{\partial}{\partial x^{i}}$, and

$$
w_{n}=\sum_{i=1}^{n}(-1)^{i-1} \frac{\partial}{\partial x^{i}} \otimes \frac{\partial}{\partial x^{1}} \wedge \frac{\partial}{\partial x^{2}} \wedge \ldots \frac{\widehat{\partial}}{\partial x^{i}} \ldots \wedge \frac{\partial}{\partial x^{n}} .
$$

Let $g_{n}^{*}=\sum_{i=1}^{n} d x^{i} \otimes d x^{i}, w_{n}^{*}=\sum_{i=1}^{n}(-1)^{n-1} d x^{i} \otimes d x^{1} \wedge d x^{2} \wedge \ldots \widehat{d x^{i}} \ldots \wedge d x^{n}$. Also from [1]

$$
\left(\mathfrak{s o}(n) \otimes J_{n}^{\wedge k}\right)^{\mathfrak{s o}(n)} \simeq \begin{cases}\left\langle s_{n}\right\rangle & k=2 \\ \left\langle\gamma_{n}\right\rangle & k=n-2 \\ 0 & \text { otherwise }\end{cases}
$$

where $s_{n}=\sum_{1 \leq i<j \leq n} \alpha_{i j} \otimes \frac{\partial}{\partial x^{i}} \wedge \frac{\partial}{\partial x^{j}}$, and

$$
\gamma_{n}=\sum_{1 \leq i<j \leq n}(-1)^{i+j-1} \alpha_{i j} \otimes \frac{\partial}{\partial x^{1}} \wedge \frac{\partial}{\partial x^{2}} \wedge \ldots \frac{\widehat{\partial}}{\partial x^{i}} \ldots \frac{\widehat{\partial}}{\partial x^{j}} \ldots \wedge \frac{\partial}{\partial x^{n}} .
$$

Let $s_{n}^{*}=\sum_{1 \leq i<j \leq n} \alpha_{i j}^{*} \otimes d x^{i} \wedge d x^{j}$, and

$$
\gamma_{n}^{*}=\sum_{1 \leq i<j \leq n} \alpha_{i j}^{*} \otimes d x^{1} \wedge d x^{2} \ldots \widehat{d x}^{i} \ldots \widehat{d x}^{j} \ldots \wedge d x^{n}
$$

In the cochain complex $\left(\operatorname{Hom}\left(\left(\mathfrak{h}_{n} \otimes J_{n}^{*}\right)^{\mathfrak{s o}(n)}, \mathbf{R}\right), \delta\right)$, we have $\delta\left(g_{n}^{*}\right)=-2 s_{n}^{*}, \delta\left(\gamma_{n}^{*}\right)=0$, $\delta\left(w_{n}^{*}\right)=0$.

Lemma 3.5. For $n \geq 3, H_{\text {Lie }}^{*}\left(J_{n} ; \mathfrak{h}_{n}^{\prime}\right) \simeq H_{\text {Lie }}^{*}(\mathfrak{s o}(n) ; \mathbf{R}) \otimes\left\langle\gamma_{n}^{*}, w_{n}^{*}\right\rangle$.

In the long exact sequence (3.2), the map $\pi_{R}^{*}: H_{\text {Lie }}^{*}\left(\mathfrak{h}_{n} ; \mathbf{R}\right) \rightarrow H_{\text {Lie }}^{*}\left(\mathfrak{h}_{n} ; \mathfrak{h}_{n}^{\prime}\right)$ sends $H_{\text {Lie }}^{*}(\mathfrak{s o}(n) ; \mathbf{R}) \otimes\left\langle v_{n}^{*}\right\rangle$ to $H_{\text {Lie }}^{*}(\mathfrak{s o}(n) ; \mathbf{R}) \otimes\left\langle w_{n}^{*}\right\rangle$. It follows that 
Lemma 3.6. For $m \geq 0$,

$$
H R^{m}\left(\mathfrak{h}_{n}\right) \simeq H_{\mathrm{Lie}}^{m+3}(\mathfrak{s o}(n) ; \mathbf{R}) \oplus\left(H_{\mathrm{Lie}}^{m+3-n}(\mathfrak{s o}(n) ; \mathbf{R}) \otimes\left\langle\gamma_{n}^{*}\right\rangle\right)
$$

where $c_{R}\left[H R^{m}\left(\mathfrak{h}_{n}\right)\right]=H_{\mathrm{Lie}}^{m+3}(\mathfrak{s o}(n) ; \mathbf{R})$ and

$$
H_{\text {Lie }}^{m+3-n}(\mathfrak{s o}(n) ; \mathbf{R}) \otimes\left\langle\gamma_{n}^{*}\right\rangle \subseteq \operatorname{Im}\left[H_{\text {Lie }}^{m+1}\left(\mathfrak{h}_{n} ; \mathfrak{h}_{n}^{\prime}\right) \rightarrow H R^{m}\left(\mathfrak{h}_{n}\right)\right]
$$

in exact sequence (3.2).

To compute $H_{\text {Lie }}^{*}\left(\mathfrak{h}_{n} ; \mathfrak{h}_{n}\right)$, we must first identify $\left[\operatorname{Hom}\left(J_{n}^{\wedge *}, \mathfrak{h}_{n}\right)\right]^{\mathfrak{s o}(n)}$.

Lemma 3.7. Let $\varphi \in \operatorname{Hom}\left(J_{n}^{\wedge k}, \mathfrak{h}_{n}\right)$ and $z=\frac{\partial}{\partial x^{i 1}} \wedge \frac{\partial}{\partial x^{2}} \wedge \ldots \wedge \frac{\partial}{\partial x^{i} k}$. There is an $\mathfrak{s o}(n)$-equivariant isomorphism

$$
\Phi: \operatorname{Hom}\left(J_{n}^{\wedge k}, \mathfrak{h}_{n}\right) \rightarrow J_{n}^{\wedge k} \otimes \mathfrak{h}_{n} \text { given by } \Phi(\varphi)=\sum_{i_{1}<i_{2}<\ldots<i_{k}} z \otimes \varphi(z) .
$$

Proof. The proof is a simple calculation.

Thus, $\left[\operatorname{Hom}\left(J_{n}^{\wedge k}, \mathfrak{h}_{n}\right)\right]^{\mathfrak{s o}(n)} \simeq\left(J_{n}^{\wedge k} \otimes \mathfrak{h}_{n}\right)^{\mathfrak{s o}(n)} \simeq\left(\mathfrak{h}_{n} \otimes J_{n}^{\wedge k}\right)^{\mathfrak{s o}(n)}$, and the latter are discussed earlier in this section.

By identification of invariants under $\Phi$, we have

Lemma 3.8. The invariants of $\left[\operatorname{Hom}\left(J_{n}^{\wedge *}, \mathfrak{h}_{n}\right)\right]^{\mathfrak{s o}(n)}, n \geq 3$, are given by

$$
\begin{aligned}
& I \in \operatorname{Hom}\left(J_{n}, \mathfrak{h}_{n}\right), \quad \rho \in \operatorname{Hom}\left(J_{n}^{\wedge 2}, \mathfrak{h}_{n}\right), \\
& \Gamma \in \operatorname{Hom}\left(J_{n}^{\wedge(n-2)}, \mathfrak{h}_{n}\right), \quad \mu \in \operatorname{Hom}\left(J_{n}^{\wedge(n-1)}, \mathfrak{h}_{n}\right),
\end{aligned}
$$

where $I\left(\frac{\partial}{\partial x^{i}}\right)=\frac{\partial}{\partial x^{i}}, i=1,2, \ldots, n, \rho\left(\frac{\partial}{\partial x^{i}} \wedge \frac{\partial}{\partial x^{j}}\right)=\alpha_{i j}$

$$
\begin{aligned}
& \Gamma\left(\frac{\partial}{\partial x^{1}} \wedge \frac{\partial}{\partial x^{2}} \wedge \ldots \frac{\widehat{\partial}}{\partial x^{i}} \ldots \frac{\widehat{\partial}}{\partial x^{j}} \ldots \wedge \frac{\partial}{\partial x^{n}}\right)=(-1)^{i+j-1} \alpha_{i j} \\
& \mu\left(\frac{\partial}{\partial x^{1}} \wedge \frac{\partial}{\partial x^{2}} \wedge \ldots \frac{\partial}{\partial x^{j}} \ldots \wedge \frac{\partial}{\partial x^{n}}\right)=(-1)^{j-1} \frac{\partial}{\partial x^{j}} .
\end{aligned}
$$

Now, $\delta I=0, \delta \rho=0$, and $\delta \Gamma=(n-1)(-1)^{n-1} \mu$.

Lemma 3.9. For $n \geq 3, H_{\text {Lie }}^{*}\left(\mathfrak{h}_{n} ; \mathfrak{h}_{n}\right) \simeq\langle I, \rho\rangle \otimes H_{\text {Lie }}^{*}(\mathfrak{s o}(n) ; \mathbf{R})$.

Note that $I: J_{n} \rightarrow \mathfrak{h}_{n}$ may be extended to $I: \mathfrak{h}_{n} \rightarrow \mathfrak{h}_{n}$ by requiring that $I\left(\alpha_{i j}\right)=0$. Also, $\rho: J_{n}^{\wedge 2} \rightarrow \mathfrak{h}_{n}$ may be extended to $\rho: \mathfrak{h}_{n}^{\wedge 2} \rightarrow \mathfrak{h}_{n}$ by requiring that $\rho\left(g_{1} \wedge g_{2}\right)=0$ if either $g_{i} \in \mathfrak{s o}(n)$. Any element $\theta \in H_{\text {Lie }}^{k}(\mathfrak{s o}(n) ; \mathbf{R})$ is represented by an $\mathfrak{s o}(n)$-invariant cocycle $\theta:(\mathfrak{s o}(n))^{\wedge k} \rightarrow \mathbf{R}$ that can be extended to $\theta: \mathfrak{h}_{n}^{\wedge k} \rightarrow \mathbf{R}$ by requiring that $\theta\left(h_{1} \wedge h_{2} \wedge \ldots \wedge h_{n}\right)=0$ if any $h_{i} \in J_{n}$. Under the isomorphism of the Hochschild-Serre spectral sequence, $I \otimes \theta$ and $\rho \otimes \theta$ correspond to the cocycles $I \wedge \theta: \mathfrak{h}_{n}^{\wedge(k+1)} \rightarrow \mathfrak{h}_{n}$ and $\rho \wedge \theta: \mathfrak{h}_{n}^{\wedge(k+2)} \rightarrow \mathfrak{h}_{n}$ respectively, where $I \wedge \theta$ and $\rho \wedge \theta$ represent the shuffle product. For completeness, we close this section with a statement about $H_{\text {Lie }}^{*}(\mathfrak{s o}(n) ; \mathbf{R})$, although the specific elements in $H_{\text {Lie }}^{*}(\mathfrak{s o}(n) ; \mathbf{R})$ do not survive to $H L^{*}\left(\mathfrak{h}_{n} ; \mathfrak{h}_{n}\right)$. From [8], we have 
Theorem 3.10. Let $n \geq 3$. For $n$ odd,

$$
H_{\text {Lie }}^{*}(\mathfrak{s o}(n) ; \mathbf{R}) \simeq \Lambda\left[\left\{x_{4 i-1} \mid 0<2 i<n\right\}\right] .
$$

For $n$ even,

$$
H_{\text {Lie }}^{*}(\mathfrak{s o}(n) ; \mathbf{R}) \simeq \Lambda\left[\left\{x_{4 i-1} \mid 0<2 i<n\right\}\right] \otimes \Lambda\left[\left\{y_{n-1}\right\}\right] .
$$

3.3. Leibniz Cohomology with Adjoint Coefficients. In this subsection we compute $H L^{*}\left(\mathfrak{h}_{n} ; \mathfrak{h}_{n}\right)$ for the affine extension $\mathfrak{h}_{n}$ of $\mathfrak{s o}(n), n \geq 3$. In low dimensions, we have

$$
\begin{aligned}
H L^{0}\left(\mathfrak{h}_{n} ; \mathfrak{h}_{n}\right) & \simeq H_{\text {Lie }}^{0}\left(\mathfrak{h}_{n} ; \mathfrak{h}_{n}\right) \simeq\left(\mathfrak{h}_{n}\right)^{\mathfrak{s o}(n)} \simeq\{0\} \\
H L^{1}\left(\mathfrak{h}_{n} ; \mathfrak{h}_{n}\right) & \simeq H_{\text {Lie }}^{1}\left(\mathfrak{h}_{n} ; \mathfrak{h}_{n}\right) \simeq\langle I\rangle
\end{aligned}
$$

where $I: \mathfrak{h}_{n} \rightarrow \mathfrak{h}_{n}$ is given above. The calculations for $H L^{*}\left(\mathfrak{h}_{n} ; \mathfrak{h}_{n}\right)$ proceed in a recursive manner, using results from lower dimensions to compute higher dimensions. Since the $H R^{m}\left(\mathfrak{h}_{n}\right)$ groups are know by Lemma (3.6), information can be gleaned about the Pirashvili spectral sequence with

$$
E_{2}^{m, k} \simeq H R^{m}\left(\mathfrak{h}_{n}\right) \otimes H L^{k}\left(\mathfrak{h}_{n} ; \mathfrak{h}_{n}\right), \quad m \geq 0, \quad k \geq 0,
$$

where previous results are substituted for $H L^{k}\left(\mathfrak{h}_{n} ; \mathfrak{h}_{n}\right)$. This spectral sequence converges to $H_{\text {rel }}^{*}\left(\mathfrak{h}_{n} ; \mathfrak{h}_{n}\right)$. The $H_{\text {rel }}^{*}\left(\mathfrak{h}_{n} ; \mathfrak{h}_{n}\right)$ groups can then be inserted into the "Lie-to-Leibniz" long exact sequence (3.1) to determine the next dimension(s) of $H L^{*}\left(\mathfrak{h}_{n} ; \mathfrak{h}_{n}\right)$. To illustrate this strategy in an easy example, note that since

$$
H L^{0}\left(\mathfrak{h}_{n} ; \mathfrak{h}_{n}\right)=0,
$$

we have $E_{2}^{0,0}=0$. (In fact, $E_{2}^{m, 0}=0, m \geq 0$ ). Thus, $H_{\text {rel }}^{0}\left(\mathfrak{h}_{n} ; \mathfrak{h}_{n}\right)=0$, and

$$
H_{\text {Lie }}^{2}\left(\mathfrak{h}_{n} ; \mathfrak{h}_{n}\right) \stackrel{\pi_{\text {rel }}^{*}}{\longrightarrow} H L^{2}\left(\mathfrak{h}_{n} ; \mathfrak{h}_{n}\right)
$$

is an isomorphism. In fact, $H L^{2}\left(\mathfrak{h}_{n} ; \mathfrak{h}_{n}\right)$ is generated by the class of

$$
\mathfrak{h}_{n} \otimes \mathfrak{h}_{n} \stackrel{\pi}{\longrightarrow} \mathfrak{h}_{n} \wedge \mathfrak{h}_{n} \stackrel{\rho}{\longrightarrow} \mathfrak{h}_{n}
$$

which we simply denote as $\rho: \mathfrak{h}_{n}^{\otimes 2} \rightarrow \mathfrak{h}_{n}$ with $\rho\left(\frac{\partial}{\partial x^{i}} \otimes \frac{\partial}{\partial x^{j}}\right)=\alpha_{i j}$ for $i=1,2, \ldots, n$, and $j=1,2, \ldots, n$. Note that $\alpha_{i j}=-\alpha_{j i}$.

Lemma 3.11. For $n \geq 3,0 \leq * \leq n+1$, we have $H L^{*}\left(\mathfrak{h}_{n} ; \mathfrak{h}_{n}\right) \simeq\langle I, \rho\rangle \otimes\left(\mathbf{R} \oplus\left\langle\gamma_{n}^{*}\right\rangle\right)$, where

$$
\gamma_{n}^{*}=\sum_{1 \leq i<j \leq n}(-1)^{i+j-1} \alpha_{i j}^{*} \otimes d x^{1} \wedge d x^{2} \wedge \ldots \widehat{d} x^{i} \ldots \widehat{d x^{j}} \ldots \wedge d x^{n}
$$


Proof. The results for $*=0,1,2$ follow from above. We consider the next iteration of elements in the $E_{2}^{*, *}$ term of the Pirashvili spectral sequence. These are:

$$
\begin{aligned}
& I \otimes \gamma_{n}^{*} \in H L^{1}\left(\mathfrak{h}_{n} ; \mathfrak{h}_{n}\right) \otimes H R^{n-3}\left(\mathfrak{h}_{n}\right) \hookrightarrow E_{2}^{n-3,1} \\
& \rho \otimes \gamma_{n}^{*} \in H L^{2}\left(\mathfrak{h}_{n} ; \mathfrak{h}_{n}\right) \otimes H R^{n-3}\left(\mathfrak{h}_{n}\right) \hookrightarrow E_{2}^{n-3,2} \\
& I \otimes \theta^{\prime} \in H L^{1}\left(\mathfrak{h}_{n} ; \mathfrak{h}_{n}\right) \otimes H R^{m}\left(\mathfrak{h}_{n}\right) \hookrightarrow E_{2}^{m, 1} \\
& \rho \otimes \theta^{\prime} \in H L^{2}\left(\mathfrak{h}_{n} ; \mathfrak{h}_{n}\right) \otimes H R^{m}\left(\mathfrak{h}_{n}\right) \hookrightarrow E_{2}^{m, 2},
\end{aligned}
$$

where $c_{R}\left(\theta^{\prime}\right)=\theta \in H_{\mathrm{Lie}}^{m+3}(\mathfrak{s o}(n) ; \mathbf{R})$ in exact sequence (3.2) (the "Lie-coadjoint" exact sequence).

Now,

$$
\begin{array}{r}
\delta\left(I \otimes \gamma_{n}^{*}\right)\left(g_{1} \otimes g_{2} \otimes \ldots \otimes g_{n+1}\right)=(\delta I) \otimes \gamma_{n}^{*}-I \otimes\left(\delta \gamma_{n}^{*}\right)+ \\
\sum_{i=3}^{n+1}(-1)^{i}\left(g_{i} I\right)\left(g_{1}\right) \gamma_{n}^{*}\left(g_{2} \otimes g_{3} \otimes \ldots \widehat{g}_{i} \ldots \otimes g_{n+1}\right) .
\end{array}
$$

Note that $\delta I=0$ and $\delta\left(\gamma_{n}^{*}\right)=0$. Since $I$ is an $\mathfrak{s o}(n)$-invariant as well as an $J_{n^{-}}$ invariant, we have $g_{i} I=0$ for all $g_{i} \in \mathfrak{h}_{n}$. Thus, $\delta\left(I \otimes \gamma_{n}^{*}\right)=0$, and $I \otimes \gamma_{n}^{*}$ is an absolute cocycle in both $C_{\text {rel }}^{n-2}\left(\mathfrak{h}_{n} ; \mathfrak{h}_{n}\right)$ and $C L^{n}\left(\mathfrak{h}_{n} ; \mathfrak{h}_{n}\right)$. Since $c_{\text {rel }}\left(I \otimes \gamma_{n}^{*}\right)=\left[\delta\left(I \otimes \gamma_{n}^{*}\right)\right]=0$ in $H_{\text {Lie }}^{n+1}\left(\mathfrak{h}_{n} ; \mathfrak{h}_{n}\right), I \otimes \gamma_{n}^{*}$ represents an element in $H L^{n}\left(\mathfrak{h}_{n} ; \mathfrak{h}_{n}\right)$. Also,

$$
\begin{aligned}
\delta\left(\rho \otimes \gamma_{n}^{*}\right) & \left(g_{1} \otimes g_{2} \otimes \ldots \otimes g_{n+2}\right)=(\delta \rho) \otimes \gamma_{n}^{*}+\rho \otimes\left(\delta \gamma_{n}^{*}\right)+ \\
& \sum_{i=4}^{n+2}(-1)^{i}\left(g_{i} \rho\right)\left(g_{1} \otimes g_{2}\right) \gamma_{n}^{*}\left(g_{3} \otimes g_{4} \otimes \ldots \widehat{g}_{i} \ldots \otimes g_{n+2}\right) .
\end{aligned}
$$

Clearly, $\delta \rho=0$ and $\delta \gamma_{n}^{*}=0$. In the last summand in equation (3.4) above, the $\gamma_{n}^{*}$ term is zero unless $g_{3} \in \mathfrak{s o}(n)$ and $g_{i} \in J_{n}, i \geq 4$. Since $d^{2}\left(\rho \otimes \gamma_{n}^{*}\right) \in F_{n-1}, d^{2}\left(\rho \otimes \gamma_{n}^{*}\right)$ is skew-symmetric in $g_{3}$ and $g_{4}$. But every term of $\gamma_{n}^{*}\left(g_{4} \otimes g_{3} \otimes \ldots \widehat{g}_{i} \ldots g_{n+2}\right)$ would then be zero. Thus, $d^{2}\left(\rho \otimes \gamma_{n}^{*}\right)=0$. The same argument applies to higher differentials such as $d^{3}\left(\rho \otimes \gamma_{n}^{*}\right) \in F_{n}$. Thus $\rho \otimes \gamma_{n}^{*}$ corresponds to a class in $H_{\text {rel }}^{n+1}\left(\mathfrak{h}_{n} ; \mathfrak{h}_{n}\right)$. Skewsymmetrization considerations of $\gamma_{n}^{*}$ also lead to $c_{\text {rel }}\left(\rho \otimes \gamma_{n}^{*}\right)=\left[\delta\left(\rho \otimes \gamma_{n}^{*}\right)\right]=0$ in $H_{\text {Lie }}^{n+2}\left(\mathfrak{h}_{n} ; \mathfrak{h}_{n}\right)$. Thus, $\rho \otimes \gamma_{n}^{*}$ corresponds to a class in $H L^{n+1}\left(\mathfrak{h}_{n} ; \mathfrak{h}_{n}\right)$.

Consider $\theta^{\prime} \in H R^{m}\left(\mathfrak{h}_{n}\right)$ with $c_{R}\left(\theta^{\prime}\right)=\theta \in H_{\text {Lie }}^{m+3}(\mathfrak{s o}(n) ; \mathbf{R})$. Then

$$
\delta\left(I \otimes \theta^{\prime}\right)=-I \otimes\left(\delta \theta^{\prime}\right)=-I \otimes c_{R}\left(\theta^{\prime}\right)=-I \otimes \theta .
$$

From the Hochschild-Serre spectral sequence, it follows that $[I \otimes \theta]=[I \wedge \theta]$ represents a non-zero class in $H_{\text {Lie }}^{*}\left(\mathfrak{h}_{n} ; \mathfrak{h}_{n}\right)$. Now, $\pi_{\text {rel }}^{*}([I \otimes \theta])=0$ in exact sequence ([3.1), since $I \otimes \theta$ is a coboundary in $C L^{*}\left(\mathfrak{h}_{n} ; \mathfrak{h}_{n}\right)$. Thus, $I \otimes \theta^{\prime}$ represents a non-zero element in $H_{\text {rel }}^{*}\left(\mathfrak{h}_{n} ; \mathfrak{h}_{n}\right)$ with

$$
c_{\mathrm{rel}}\left(\left[I \otimes \theta^{\prime}\right]\right)=\left[\delta\left(I \otimes \theta^{\prime}\right)\right]=[-I \otimes \theta] .
$$


Now,

$$
\begin{aligned}
\delta\left(\rho \otimes \theta^{\prime}\right) & \left(g_{1} \otimes g_{2} \otimes \ldots \otimes g_{m+5}\right)=\rho \otimes\left(\delta \theta^{\prime}\right)+ \\
& \sum_{j=4}^{m+5}(-1)^{j}\left(g_{i} \rho\right)\left(g_{1} \otimes g_{2}\right) \theta^{\prime}\left(g_{3} \otimes g_{4} \otimes \ldots \hat{g}_{j} \ldots \otimes g_{m+5}\right)
\end{aligned}
$$

The element $\theta^{\prime} \in H R^{m}\left(\mathfrak{h}_{n}\right)$ can be chosen so that $\theta^{\prime}\left(y_{1} \otimes y_{2} \otimes \ldots \otimes y_{m+2}\right)=0$ if any $y_{i} \in J_{n}$, while $\rho$ is an $\mathfrak{s o}(n)$-invariant. Suppose then that $g_{j} \in J_{n}$ for one and only one $j \in\{4,5, \ldots, m+5\}$. We have $\rho \otimes \theta^{\prime} \in F_{m}$ and $d^{2}\left(\rho \otimes \theta^{\prime}\right) \in F_{m+2}$. Clearly, $\delta\left(\theta^{\prime}\right)=\theta$ is already skew-symmetric in $g_{3}, g_{4}, \ldots, g_{m+5}$ in equation (3.5). Thus, for $d^{2}\left(\rho \otimes \theta^{\prime}\right)$, we must have

$$
\begin{aligned}
& \left(g_{j} \rho\right)\left(g_{1} \otimes g_{2}\right) \theta^{\prime}\left(g_{3} \otimes g_{4} \otimes \ldots \widehat{g}_{j} \ldots \otimes g_{m+5}\right)= \\
& \quad \pm\left(g_{3} \rho\right)\left(g_{1} \otimes g_{2}\right) \theta^{\prime}\left(g_{j} \otimes g_{4} \otimes \ldots \widehat{g}_{j} \ldots \otimes g_{m+5}\right)=0
\end{aligned}
$$

since $g_{3} \in \mathfrak{s o}(n)$ and $\rho$ is an $\mathfrak{s o}(n)$-invariant. Also,

$$
d^{3}\left(\rho \otimes \theta^{\prime}\right) \in H L^{0}\left(\mathfrak{h}_{n} ; \mathfrak{h}_{n}\right) \otimes H R^{m+3}\left(\mathfrak{h}_{n}\right)=\{0\} .
$$

Thus, $\rho \otimes \theta^{\prime}$ represents an element in $H_{\text {rel }}^{m+2}\left(\mathfrak{h}_{n} ; \mathfrak{h}_{n}\right)$ and

$$
c_{\text {rel }}\left(\left[\rho \otimes \theta^{\prime}\right]\right)=\left[\delta\left(\rho \otimes \theta^{\prime}\right)\right]=[\rho \otimes \theta]=[\rho \wedge \theta]
$$

in $H_{\text {Lie }}^{m+5}\left(\mathfrak{h}_{n} ; \mathfrak{h}_{n}\right)$, where $c_{\text {rel }}$ is the connecting homomorphism the "Lie-to-Leibniz" exact sequence.

Theorem 3.12. For $n \geq 3, H L^{*}\left(\mathfrak{h}_{n} ; \mathfrak{h}_{n}\right) \simeq\langle I, \rho\rangle \otimes T\left(\gamma_{n}^{*}\right)$, where $T\left(\gamma_{n}^{*}\right):=\sum_{q \geq 0}\left\langle\gamma_{n}^{*}\right\rangle \otimes q$ is the tensor algebra on the class of $\gamma_{n}^{*}$.

Proof. The result for $q=0$ and $q=1$ follows from Lemma (3.11). We begin with the next iteration of elements in the $E_{2}^{*, *}$ term of the Pirashvili spectral sequence. Consider

$$
\left(I \otimes \gamma_{n}^{*}\right) \otimes \theta^{\prime} \in H L^{n}\left(\mathfrak{h}_{n} ; \mathfrak{h}_{n}\right) \otimes H R^{m}\left(\mathfrak{h}_{n}\right) \subseteq E_{2}^{m, n}
$$

with $c_{R}\left(\theta^{\prime}\right)=\theta \in H_{\text {Lie }}^{m+3}(\mathfrak{s o}(n) ; \mathbf{R})$. Then

$$
\begin{aligned}
& \delta\left(\left(I \otimes \gamma_{n}^{*}\right) \otimes \theta^{\prime}\right)\left(g_{1} \otimes \ldots \otimes g_{n+m+3}\right)=(-1)^{n}\left(I \otimes \gamma_{n}^{*}\right) \otimes\left(\delta \theta^{\prime}\right)+ \\
& \quad \sum_{j=n+2}^{n+m+3}(-1)^{j}\left(g_{j}\left(I \otimes \gamma_{n}^{*}\right)\right)\left(g_{1} \otimes \ldots \otimes g_{n}\right) \theta^{\prime}\left(g_{n+1} \otimes \ldots \widehat{g}_{j} \ldots \otimes g_{n+m+3}\right) .
\end{aligned}
$$

Now, $I$ is an $\mathfrak{h}_{n}$-invariant, and it can be checked that $\gamma_{n}^{*}$ is as well. Thus, $I \otimes \gamma_{n}^{*}$ is an $\mathfrak{h}_{n}$-invariant and $g_{j}\left(I \otimes \gamma_{n}^{*}\right)=0$ for all $g_{j} \in \mathfrak{h}_{n}$. Thus,

$$
\begin{aligned}
\delta\left(\left(I \otimes \gamma_{n}^{*}\right) \otimes \theta^{\prime}\right) & =(-1)^{n}\left(I \otimes \gamma_{n}^{*}\right) \otimes \theta \\
& =(-1)^{n} I \otimes\left(\gamma_{n}^{*} \otimes \theta\right) \in H L^{1}\left(\mathfrak{h}_{n} ; \mathfrak{h}_{n}\right) \otimes H R^{n+m}\left(\mathfrak{h}_{n}\right) .
\end{aligned}
$$


In the Pirashvili spectral sequence, $d^{n}\left(\left(I \otimes \gamma_{n}^{*}\right) \otimes \theta^{\prime}\right)=I \otimes\left(\gamma_{n}^{*} \otimes \theta\right)$.

Now consider an $\mathfrak{s o}(n)$-invariant representation for the cohomology class corresponding to $\left[\rho \otimes \gamma_{n}^{*}\right] \in H L^{n+1}\left(\mathfrak{h}_{n} ; \mathfrak{h}_{n}\right)$. Let $\theta^{\prime} \in H R^{m}\left(\mathfrak{h}_{n}\right)$ with $c_{R}\left(\theta^{\prime}\right)=\theta \in$ $H_{\text {Lie }}^{m+3}(\mathfrak{s o}(n) ; \mathbf{R})$. By a skew symmetry argument as before, we have

$$
d^{n}\left(\left(\rho \otimes \gamma_{n}^{*}\right) \otimes \theta^{\prime}\right)=\rho \otimes\left(\gamma_{n}^{*} \otimes \theta\right)
$$

in the Pirashvili spectral sequence. For

$$
\left[\rho \otimes \gamma_{n}^{*}\right] \otimes \gamma_{n}^{*} \in H L^{n+1}\left(\mathfrak{h}_{n} ; \mathfrak{h}_{n}\right) \otimes H R^{n-3}\left(\mathfrak{h}_{n}\right) \subseteq E_{2}^{n-3, n+1}
$$

we have

$$
\begin{gathered}
\delta\left(\left[\rho \otimes \gamma_{n}^{*}\right] \otimes \gamma_{n}^{*}\right)\left(g_{1} \otimes \ldots \otimes g_{2 n+1}\right)=\delta\left(\left[\rho \otimes \gamma_{n}^{*}\right]\right) \otimes \gamma_{n}^{*}+(-1)^{n+1}\left[\rho \otimes \gamma_{n}^{*}\right] \otimes\left(\delta \gamma_{n}^{*}\right)+ \\
\sum_{j=n+3}^{2 n+1}\left(g_{j}\left[\rho \otimes \gamma_{n}^{*}\right]\left(g_{1} \otimes \ldots \otimes g_{n+1}\right)\right) \gamma_{n}^{*}\left(g_{n+2} \otimes \ldots \widehat{g}_{j} \ldots \otimes g_{2 n+1}\right)= \\
\sum_{j=n+3}^{2 n+1}\left(g_{j}\left[\rho \otimes \gamma_{n}^{*}\right]\left(g_{1} \otimes \ldots \otimes g_{n+1}\right)\right) \gamma_{n}^{*}\left(g_{n+2} \otimes \ldots \widehat{g}_{j} \ldots \otimes g_{2 n+1}\right) .
\end{gathered}
$$

Since $d^{2}\left(\left[\rho \otimes \gamma_{n}^{*}\right] \otimes \gamma_{n}^{*}\right) \in F_{n-1}, d^{2}\left(\left[\rho \otimes \gamma_{n}^{*}\right] \otimes \gamma_{n}^{*}\right)$ is skew-symmetric in the variables $g_{n+2}, g_{n+3}, \ldots, g_{2 n+1}$. By a similar argument used for $\rho \otimes \gamma_{n}^{*}$, we have that $\left[\rho \otimes \gamma_{n}^{*}\right] \otimes \gamma_{n}^{*}$ corresponds to an element in $H L^{2 n}\left(\mathfrak{h}_{n} ; \mathfrak{h}_{n}\right)$.

For $\left[I \otimes \gamma_{n}^{*}\right] \in H L^{n}\left(\mathfrak{h}_{n} ; \mathfrak{h}_{n}\right)$ and $\gamma_{n}^{*} \in H R^{n-3}\left(\mathfrak{h}_{n}\right)$, we have

$$
\begin{array}{r}
\delta\left(\left[I \otimes \gamma_{n}^{*}\right] \otimes \gamma_{n}^{*}\right)\left(g_{1} \otimes \ldots \otimes g_{2 n}\right)=\delta\left(I \otimes \gamma_{n}^{*}\right) \otimes \gamma_{n}^{*}+(-1)^{n}\left(I \otimes \gamma_{n}^{*}\right) \otimes\left(\delta \gamma_{n}^{*}\right)+ \\
\sum_{j=n+2}^{2 n}(-1)^{j}\left(g_{j}\left(I \otimes \gamma_{n}^{*}\right)\right)\left(g_{1} \otimes \ldots \otimes g_{n}\right) \gamma_{n}^{*}\left(g_{n+1} \otimes \ldots \widehat{g}_{j} \ldots g_{2 n}\right)=0
\end{array}
$$

since $I \otimes \gamma_{n}^{*}$ is an $\mathfrak{h}_{n}$-invariant. Thus, $\left(I \otimes \gamma_{n}^{*}\right) \otimes \gamma_{n}^{*}$ represents a class in $H L^{2 n-1}\left(\mathfrak{h}_{n} ; \mathfrak{h}_{n}\right)$. The theorem holds for $q=2$. Also, in the Pirashvili spectral sequence, we have

$$
\begin{aligned}
& d^{n}\left(\left(I \otimes\left(\gamma_{n}^{*}\right)^{\otimes 2}\right) \otimes \theta^{\prime}\right)=\left(I \otimes \gamma_{n}^{*}\right) \otimes\left(\gamma_{n}^{*} \otimes \theta\right) \\
& d^{n}\left(\left(\rho \otimes\left(\gamma_{n}^{*}\right)^{\otimes 2}\right) \otimes \theta^{\prime}\right)=\left(\rho \otimes \gamma_{n}^{*}\right) \otimes\left(\gamma_{n}^{*} \otimes \theta\right) .
\end{aligned}
$$

By induction on $q, H L^{*}\left(\mathfrak{h}_{n} ; \mathfrak{h}_{n}\right)$ is the direct sum of vector spaces $\langle I, \rho\rangle \otimes\left(\gamma_{n}^{*}\right)^{\otimes q}$. We conclude that

$$
H L^{*}\left(\mathfrak{h}_{n} ; \mathfrak{h}_{n}\right) \simeq\langle I, \rho\rangle \otimes T\left(\gamma_{n}^{*}\right)
$$




\section{REFERENCES}

[1] Biyogman, G. R., "On the Leibniz (Co)homology of the Lie Algebra of the Euclidean Group," Journal of Pure and Applied Algebra, Vol. 215 (2011), pp. 1889-1901.

[2] Bloh, A., "On a Generalization of a Concept of Lie Algebras," Dokl. Akad. Nauk., SSSR, Vol. 165 (1965), pp. 471-473.

[3] do Carmo, M.P., Riemannian Geometry, Flaherty, F., translator, Birkhäuser, Basel, 1992.

[4] Fialowski, A., Mandal, A. "Leibniz Deformations of a Lie Algebra," Journal of Mathematical Physics, Vol. 49, 9 (20008), doi.org/10.1063/1.2981562.

[5] Fialoswki, A., Mandal, A., Mukherjee, G., "Versal Deformations of Leibniz Algebras," KTheory, Vol. 3, 2 (2009), pp. 327-358.

[6] Feldvoss, J., Wagemann, F., "On Leibniz Cohomology," Journal of Algebra, Vol. 569 (2021), pp. 276-317.

[7] Hochschild, G., Serre, J-P., "Cohomology of Lie Algebras," Annals of Mathematics, Vol 57, 3 (1953), pp. 591-603.

[8] Ito, K., Nihon, S., Encyclopedic Dictionary of Mathematics, MIT Press, Cambridge, Mass., 1987.

[9] Loday, J.-L., Cyclic Homology, Spring Verlag, Heidelberg, 1992.

[10] Loday, J.-L., Pirashvili, T., "Universal Enveloping Algebras of Leibniz Algebras and (Co)homology," Math. Ann., Vol. 296 (1993), pp. 139-158.

[11] Lodder, J. "Leibniz Cohomology for Differentiable Manifolds," Ann. Institut Fourier (Grenoble), Vol. 48, 1 (1998), pp. 73-95.

[12] Pirashvili, T., "On Leibniz Homology," Ann. Institut Fourier (Grenoble), Vol. 44, 2 (1994), pp. 401-411.

[13] Sakai, T., Riemannian Geometry, Translations of Mathematical Monographs, Vol. 149, American Mathematical Society, Providence, RI, 1996. 